\title{
Chaos in Josephson Junctions and SQUIDs
}

\author{
N.F. Pedersen, Lyngby
}

(The Technical University of Denmark)

Josephson junctions are among the most attractive non-linear dynamical systems for the study of chaos. The reason is that on the one hand the system is sufficiently non-linear to display almost all known types of complicated chaotic behaviour, and yet, the underlying equation of motion is the pendulum equation, which is quite simple and well known to most scientists. The simplicity of the pendulum model also offers a means of visualizing the complicated dynamical behaviour; many scientists argue over the behaviour of Josephson junctions by rotating their arm as a pendulum, as may be observed in many conferences.

Second, although the model equations are simple, they are known to describe real Josephson junctions very well, as has been demonstrated in numerous experiments.

Finally, there are many potential applications of Josephson junctions within such diverse fields as microwave detectors, computers, magnetometers etc. The newly discovered high transition temperature ceramic oxide superconductors give promise of many further ap-
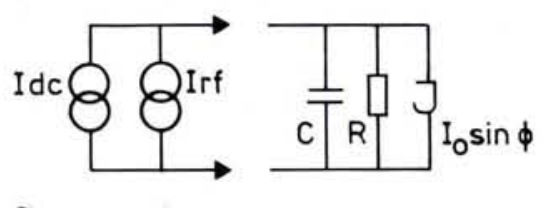

a

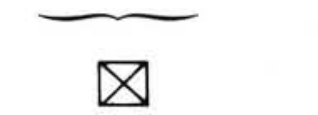

b

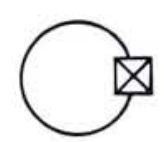

C

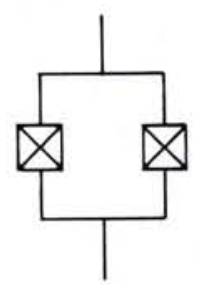

Fig. 1 - a) Equivalent diagram for the RFdriven Josephson junction; b) RF SQUID; c) DC SQUID. plications; since the new superconductors need only liquid nitrogen for cooling they have enhanced the technological importance of Josephson junction devices and suggest a much wider use than has been known until today. As I hope to clarify in the following, several of the unique applications of Josephson junction devices involve parameter ranges that are close to those where chaos occurs. Hence, the study of chaos in such systems is of the utmost importance for applications. A severe complication is that the magnitude of "chaos noise" may be comparable to that of thermal noise; further the interaction between the two noise components is highly non-linear, and may be extremely complicated.

\section{The Josephson Junction}

A Josephson junction consists of two superconductors separated by a thin insulating barrier. The important variable is the difference of the quantum mechanical phase between the two sides, $\phi=$ $\phi_{2}-\phi_{1}$. Physically the Josephson junction may take the shape of a sandwich type thin film component, a point contact or a narrow constriction. The materials used are typically metals such as niobium, lead, tin etc. with their natural oxides used as the insulating barrier. The future will most likely also show the use of the new high transition temperature ceramic superconductors, in Josephson junction circuits.

The most widely investigated Josephson junction system with possible chaotic solutions is the radio frequency (RF) current driven junction, a description of which may be found for example in the recent book by Barone and Paterno [1]. Chaotic solutions are derived for certain parameter ranges, and the literature on this particular system has grown steadily, the contributions consisting mostly of papers on numerical simulations. The equation of motion for the system also describes other important physical systems, such as synchronous motors, phased-locked loops, pinned charge density waves, and the periodically forced pendulum.
The equations for a current driven Josephson junction with capacitance $C$, resistance $R$, and critical current $I_{0}$ is derived from Kirchhoffs law (Fig. 1a) and may be written

$$
\begin{aligned}
C d V / d t & +V / R+I_{0} \sin \phi \\
& =I_{\mathrm{DC}}+I_{\mathrm{RF}} \sin \omega t \\
d \phi / d t & =2 \mathrm{eV} / \hbar .
\end{aligned}
$$

Here $I_{\mathrm{DC}}$ and $I_{\mathrm{RF}}$ are the DC and RF bias currents, respectively, and $\phi$ is the superconducting pair phase difference across the junction. The last equation is the famous Josephson voltage to frequency relation. These equations may be combined into a single dimensionless equation with time normalized to the reciprocal plasma frequency

$$
1 / \omega_{0}=\left(2 e I_{0} / \hbar C\right)^{-1 / 2}
$$

and current normalized to the critical current $I_{0}$ [1]. (In the corresponding pendulum system the plasma frequency corresponds to the small amplitude oscillation frequency and the critical current corresponds to the maximum gravitational torque). In the resulting equation:

$$
\begin{gathered}
d^{2} \phi / d t^{2}+\left(1 / \sqrt{ } \beta_{\mathrm{c}}\right) d \phi / d t+\sin \phi \\
=\rho+\rho_{1} \sin \Omega t
\end{gathered}
$$

where $\beta_{c}$ is the so-called McCumber damping parameter given by

$$
\beta_{\mathrm{c}}=2 e l_{\mathrm{o}} R^{2} C / \hbar \text {, }
$$

$\Omega$ is the applied frequency normalized to the plasma frequency, $\rho$ is the normalized DC bias current, and $\rho_{1}$ is the normalized microwave current amplitude.

An RF SQUID is a Josephson junction shunted with a superconducting shunt as shown in Fig. 1b. (SQUID is short for Superconducting QUantum Interference Device.) A DC SQUID consists of two Josephson junctions connected in parallel by a superconducting loop as shown in Fig. 1c. These systems give rise to non-linear equations somewhat more complicated than Eq. (2), since third and fourth order derivatives appear; they also have a variety of chaotic solutions.

Besides being a model system for chaos, Josephson junctions and SQUIDs have several potential applications such as low noise microwave parametric amplifiers, digital electronics, and sensitive magnetometers. To exploit these 


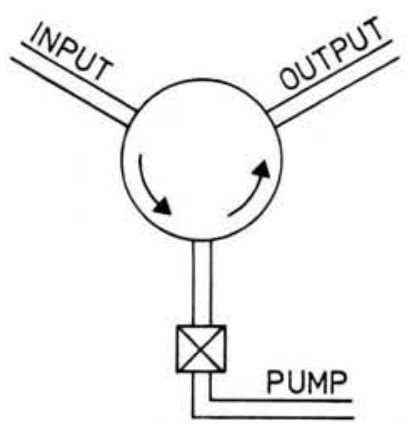

Fig. 2 - The circulator-mounted Josephson junction parametric amplifier.

applications it is often necessary to use parameter ranges close to those that make the relevant equations chaotic. Hence, investigations of chaos in Josephson junctions and SQUIDs often serve a very practical purpose.

Noise Rise and the Parametric Amplifier

As an example we shall consider the Josephson junction microwave parametric amplifier in this section. Fig. 2 shows a schematic diagram of such a circulator mounted negative impedance reflection amplifier. The signal from the input line follows the arrow to the junction, from where it is reflected to the output line (along the second arrow). If the junction has a negative resistance, the reflection coefficient is larger than one and amplification occurs.

By now such amplifiers at microwave frequencies have been fabricated with the lowest noise temperature known. This has become possible by using our knowledge of chaos and how to avoid it, as obtained from numerical simulations. However, the work leading to that result has not been trivial.

For many years experimentalists working with Josephson junction parametric amplifiers had been bothered by the so-called "noise rise", which for reasons that were not understood, sometimes appeared and manifested itself as an extremely high noise temperature (say $10^{5}-10^{6} \mathrm{~K}$ ). Traditional noise sources such as thermal noise or shot noise can only account for a noise temperature of a few $10^{3} \mathrm{~K}$ at most. It was suggested by Huberman et al. [2] in 1980 that deterministic chaos might be the origin. Now it appears that there is some truth in the idea [3], but that interaction with thermal noise is essential.

For a so-called three photon parametric amplifier (in which the pump frequency is twice the signal frequency) the problem was investigated by careful simulation and numerical calculations by Miracky et al. [4]. The authors drew the conclusion that noise in this amplifier cannot arise solely from determini- stic chaos: To achieve gain the amplifier must be operated at a stable bias point below the threshold for the period doubling bifurcation, and thus below the threshold for the onset of chaos. The observed noise rise requires the presence of thermal noise. The noise rise originates in a thermally induced hopping between a bias point in the high gain region (that would be stable in the absence of thermal noise) and an unstable point in the bifurcated region. Such hopping typically gives a very high noise temperature and enhances the low frequency part of the spectrum as will be discussed in the next section.

In the recently developed Josephson junction parametric amplifiers, where the noise rise is avoided, the junction is stabilized at low frequencies by a low resistance or superconducting shunt; hence the amplifier is conceptually similar to the RF SQUID as shown in Fig. 1b. Noise temperatures of a few Kelvin with $10 \mathrm{~dB}$ of gain can now be obtained at $10 \mathrm{GHz}$.

\section{SQUIDs and $1 / f$ noise}

In SQUIDs (Fig. 1b, c) the problem of $1 / f$ noise is of great importance, as it is in other Josephson junction devices. 1/f noise exists in many physical systems, although the origin of it is not understood in most cases. The possibility of a connection between $1 / f$ noise and chaos - possibly thermally induced - has been suggested and deserves attention. The particular type of chaos possibly responsible for low frequency excess noise is often referred to as intermittency.

Intermittency refers to qualitative changes in the state of a system on time scales much longer than the usual characteristic times of the system. Several categories of intermittency have been identified in the RF-driven Josephson junction and the SQUID. For both intrinsic systems (without external noise) and extrinsic systems (with external noise) some of those modes of intermittency may lead to a low frequency spectrum that scales approximately as $1 / f$ over several decades in frequency. As an example for the intrinsic (noise free) system, the so-called Pomeau-Manneville type 3 intermittency is generated by phase space trajectories that diverge slowly from a marginally unstable periodic orbit; the corresponding power spectrum is close to $1 / f$. As another example, noise induced (extrinsic) intermittency occurs when a system is knocked between different (possibly chaotic) attractors by an external noise source (e.g. thermal noise). An attractor is a point (for periodic solutions) or a region (for chaotic solutions) in phase space to which trajectories converge and in which they remain trapped. In the latter case it is often denoted a strange attractor. Such noise induced intermittency may also lead approximately to $1 / f$ noise spectrum with large amplitude.

A particular illustrative example of noise-induced intermittency is the case in which there are fractal basin boundaries, as has also been found for certain parameter ranges in Josephson junction systems. Imagine for example two attractors in the phase plane defined by the variables $(\phi, \dot{\phi})$ from Eq. (2). If initial conditions in the phase plane, that eventually settles on one attractor are shown in black, and initial conditions ending on the other attractor are shown in white, then the fractal basin boundaries in the phase plane will appear as alternating stripes (regions) of black and white on any length scale. Quite obviously with such fractal basin boundaries small amounts of thermal noise can kick the system from one attractor to the other, resulting in large amplitude $1 / f$ noise.
Fig. 3 - Cell to cell mapping. Big solid circles: attractor found by cell mapping; small solid circle: basin of attraction for the attractor. Curve: the strange attractor found by direct numerical integration.

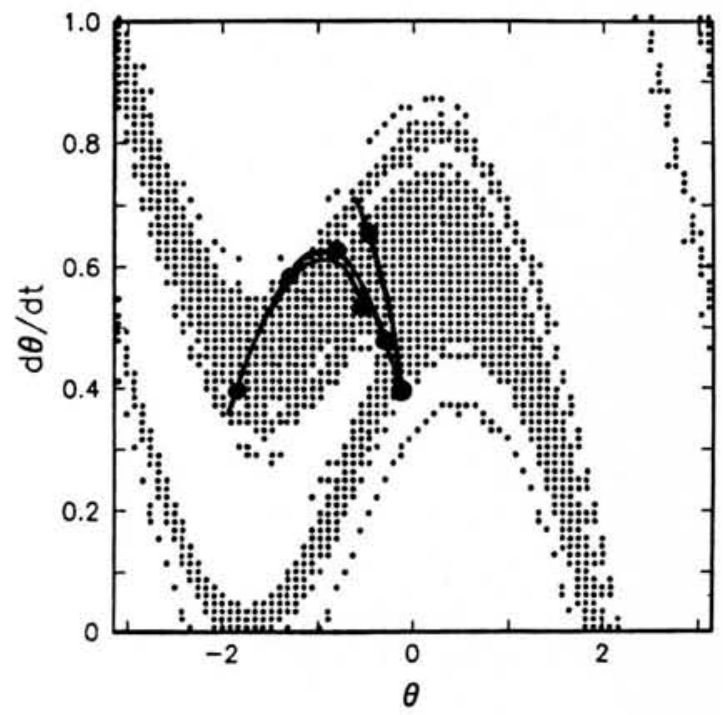




\section{Cell Mapping}

The above discussion emphasizes the importance of investigating the global behaviour of the system equation (for example Eq. (2)), i.e. with initial conditions everywhere in the phase plane. With each initial condition the system may - depending on the damping parameter - require the integration of typically 500-1000 RF cycles before the steady state is obtained with certainty; furthermore, since a reasonable coverage of initial conditions typically requires a $100 \times 100$ element grid, the computer time for such an investigation becomes very expensive indeed.

A solution to this problem recently proposed is a method called cell to cell mapping [5]. In that method the phase plane is divided into, for example, a two dimensional $100 \times 100$ grid of cells. With given initial conditions in the centre of one cell, say $(i, j)$, the system is integrated for one RF cycle and the final coordinates are noted. These coordinates belong to another cell which we denote $(m, n)$. Thus we have defined a mapping of cell $(i, j)$ on to cell $(m, n)$. The mapping of all cells in the grid is investigated that way, each cell requiring the integration of one RF cycle. The rest of the analysis is sorting, a process that is relatively inexpensive in computer time. For example fixpoints (periodic solutions) are found by asking if some cells map onto themselves (possibly after several iterations). The corresponding basin of attraction for each fixpoint is found by tracing backwards the iterations leading to the fixpoint. Specially interesting regions in phase space can be investigated by doing a local cell mapping over a smaller region.

Fig. 3 shows as an example a cell mapping computed with a $75 \times 75$ grid of cells. The parameters are chosen such that the system is chaotic with a strange attractor in the phase plane. As demonstrated in the figure, the (period 7) attractor found by cell mapping provides an approximation to the strange attractor which has been obtained by direct numerical calculation. The basin found by cell mapping is almost identical to the basin of attraction for the strange attractor. Thus the cell mapping method gives a quick overview of the global behaviour of the system, with a minimum of computer time.

Other phenomena that require knowledge about the different attractors and their basins of attraction are the socalled crises, of which an interior crisis and a boundary crisis have been clearly identified in Josephson junction systems.
A crisis results when increasing a system parameter to a critical value causes a strange attractor to collide with an unstable periodic orbit in phase space. It is called a boundary crisis if the chaotic attractor disappears as a result of the collision. Instead it may undergo a sudden expansion, in which case it is referred to as an interior crisis. A crisis may also cause intermittency and thus give rise to approximately $1 / f$ noise behaviour. Crises can be observed directly by a simple measurement of the currentvoltage curve of a Josephson junction irradiated with microwaves. For example, the interior crisis typically manifests itself as a gradual loss of phaselock on the so-called RF-induced current steps at harmonics of the voltage $\hbar \omega / 2 \mathrm{e}$. Since the voltage of these steps are used as a voltage standard with a precision of about $10^{-7}$, any small loss of phaselock will have dire consequences. Thus any voltage standard based on Josephson junctions must be carefully dimensioned so as to avoid chaos. For the new types of voltage standards with many (hundreds of) series connected junctions, this is a non-trivial problem.

\section{Conclusion}

As I hope to have demonstrated, the Josephson junction is a marvellous device with many practical applications. It is at the same time a model system for all types of chaos. Many practical applications typically involve operating close to the onset of chaos, and the detailed understanding of chaos in Josephson junctions is thus important. Since "chaotic noise" and thermal noise have

\section{Anniversary Seminar}

In honour of the Past Presidents and the Honorary Members of EPS, the 20th anniversary of the founding of the Society and the 90th anniversary of the founding of the Italian Physical Society are being celebrated in a Seminar on "Past and Future of Physics in Europe", appropriately in Pisa on 18/19 June 1988. The meeting is open to EPS Members; no registration fee will be charged.

The Seminar which follows a meeting of the EPS Executive Committee is being organized by the new EPS President, Professor R.A. Ricci (see page 56). Further information may be obtained from him through the EPS Secretariat in Geneva.

approximately the same amplitude, and since their interaction is highly nonlinear, such an understanding is by no means trivial to obtain.

\section{REFERENCES}

[1] Barone A. and Paterno G., Physics and applications of the Josephson effect (WileyInterscience, New York) 1982.

[2] Huberman B.A., Crutchfield J.P. and Packhard N.H., Appl. Phys. Lett. 37 (1980) 750.

[3] Pedersen N.F. and Davidson A., Appl. Phys. Lett. 39 (1981) 830.

[4] Miracky R.F. and Clarke J., Appl. Phys. Lett. 43 (1983) 508.

[5] Hsu C.S., Cell to Cell Mapping, Applied Mathematical Sciences 64 (Springer Verlag, Berlin) 1987.

\section{$\overline{\bar{E}} \overline{\overline{0}} \overline{\bar{E}}$ Zurich Research Laboratory 豆严皘 (Switzerland)}

The Laser Science and Technology Department of the IBM Zurich Research Laboratory in Switzerland has openings for

\section{Research Scientists (permanent research staff member position)}

to work on physics and materials science aspects of semiconductor lasers. Prospective candidates holding a Ph.D. in physics, electrical engineering or material sciences, and experience in one or more of the following areas is essential:

- Semiconductor Lasers / Optoelectronics

- III/V semiconductor processing (RIE, RIBAE, ion beam technology)

- Epitaxial material growth techniques (OMVPE, MBE, CBE)

- Defect characterization / failure analysis in III/V devices

- Design, modelling and characterization OE devices

Qualified candidates, interested in contributing to science and technology of new semiconductor lasers for computer applications, are invited to send their resumé containing curriculum vitae, a list of publications, and three letters of reference in confidence to:

IBM Research Division, Zurich Research Laboratory Säumerstrasse 4, CH-8803 Rüschlikon / Switzerland Phone: + 41 (1) 7248355 